\title{
Point Processing Method for Improving Dental Radiology Image Quality
}

\author{
Retno Supriyanti ${ }^{1}$, Ariep Soelaiman Setiadi ${ }^{1}$, Yogi Ramadhani ${ }^{1}$, Haris B. Widodo ${ }^{2}$ \\ ${ }^{1}$ Departement of Electrical Engineering, Jenderal Soedirman University, Indonesia \\ ${ }^{2}$ Departement of Dentistry, Jenderal Soedirman University, Indonesia
}

\begin{tabular}{l} 
Article Info \\
\hline Article history: \\
Received Jan 21, 2016 \\
Revised Mar 29, 20 \\
Accepted Apr 10, 201 \\
\hline Keyword: \\
Contrast stretching \\
Dentistry \\
Panoramic image \\
Point processing \\
Radiology field
\end{tabular}

\section{Corresponding Author:}

Retno Supriyannti, Departement of Electrical Engineering, Jenderal Soedirman University, Kampus Blater, Jl. Mayjend Sungkono KM 5, Blater, Purbalingga, Indonesia Email: retno_supriyanti@unsoed.ac.id

\begin{abstract}
Radiology field is very important in today's world, especially in the field of medicine including dentistry. Radiology equipment that is popular in dentistry is the panoramic machine. A panoramic image facilitate the dentist in making a diagnosis of the abnormality in the mouth and teeth. But unfortunately, for developing countries like Indonesia, panoramic machine available are low resolution which have an effect on the resulting image also has low quality. This research aims to improve the quality of the panoramic image to have a better quality. We use point processing method with emphasis on contrast stretching method. We chose this method because it is quite simple but has a high performance. Based on the second opinion from the hospital, the performance is $83.9 \%$, therefore this method is promising to be implemented on the improvement of dental radiology images.
\end{abstract}

Copyright (C) 2016 Institute of Advanced Engineering and Science. All rights reserved.

\section{INTRODUCTION}

More than a century dentistry profession using radiographic examination as a means to obtain diagnostic information cannot be obtained from the clinical examination and other tests before. Until now, dental radiography become one of the important tools used in the treatment of modern dentistry. Shooting good dental radiographic projections both intra oral and extra-oral almost a common procedure performed by a dentist in assisting the management of a case.

The usefulness of Dental Radiography of which is to: (1) Radio diagnostics / Rontgen Diagnosis: used to diagnose such abnormalities apical or per apical not detected clinically, abnormalities in the jaw, fracture of the jaw or tooth root and hidden caries (the proximal or root caries) secondary caries, caries incipient, the depth of caries and others. (2) Treatment Plan: assist in making or determining a treatment plan, such as the determination of the location of the pins or implants, condition of the root canal, determination of the type and technique (3) Supporting Treatment: help facilitate the conduct of a treatment, such as postoperative complications, treatment endodontic (4) evaluation of Care: used to evaluate the success or progress or control treatment (5) Radiography is one of the medical record that is very important and also for (6) Interest forensic [1].

Based on usability, it can be said that the Dental Radiography plays an important role in various matters in the field of dentistry. One of the tools commonly used in dental radiography is panoramic machine [2]. Refers to the advantages of using dental radiography, it is now almost all dentists use them for the benefit of their diagnosis. We have to remember that the images produced by dental radiography has qualities that are highly dependent on the resolution of which is owned by the radiology machine. Specifically for dental radiology, the equipment used is dental panoramic machine as shown in Figure 1. 


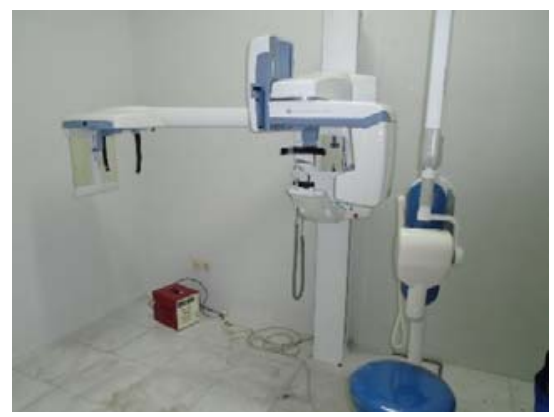

Figure 1. An example of panoramic machine

In 1995, Molander [3] did a research about image qualities in panoramic radiography. In principle, panoramic radiography is divided into two classifications that conventional and digital. Conventional panoramic has a lower resolution than the digital panoramic. While in reality, particularly in developing countries such as Indonesia, panoramic radiography available is the conventional type and the number is limited. Low resolution image will affect to the accuracy of diagnosis. This paper will discuss about simple method for improving image quality in low-resolution panoramic radiography.

According to the research about panoramic radiography, Rushton [4] did a research to identify the radiological findings from routine screening panoramic radiographs taken of adult patients in general dental practice. However his study did not provide evidence to support the practice of routine panoramic radiography of all new adult patients. Chaffin [5] did a research to examine the validity of classifying Initial Entry Training (IET) soldiers into dental fitness classification based solely on examining panoramic radiographs. Takahashi [6] investigated the detectability of the mandibular canal on dental CT reformatted images of patients before implant operation.

The detectability was significantly higher in panoramic views than in paraxial views. Suomalainen [7] cone beam computed tomography (CBCT) devices in order for improving quality of panoramic radiology. Sezgin [8] did a research to compare the effective organ doses from cone beam computed tomography (CBCT), multislice computed tomography (MSCT), and panoramic radiography. Ertas [9] presented a radiological findings and follow-up of three patients with severe atherosclerosis that was incidentally detected on Panoramic Radiology (PRs), and discuss the role and importance of PRs in the detection of carotid artery calcifications. Rotondi [10] proposed a technique that can be used to design a study measuring interobserver agreement with any number of outcomes and any number of raters. According to these research, it looks that these kind of research emphasize on the function of these kind of medical devices such as carried out by Kao [11] that proposed a fully automatic abdominal fat segmentation system for quantifying abdominal fat, including subcutaneous adipose tissue and visceral adipose tissue. Also Chang [12] presented a data hiding method to conceal secret data into color BTC compression code by rearranging high mean and low mean encoding sequences.

The proposed method is attempting to embed more secret data into the color BTC compression code. Some research that discussed about image processing are research did by Gopinathan [13] that proposed a stationary and discrete wavelet based image denoising scheme and an FFTbased image denoising scheme to remove Gaussian noise. In the first approach, high subbands are added with each other and then soft thresholding is performed. The sum of low subbands is filtered with either piecewise linear (PWL) or Lagrange or spline interpolated PWL filter. In the second approach, FFT is employed on the noisy image and then low frequency and high frequency coefficients are separated with a specified cutoff frequency. Faridah [14] discussesed the application of Snake to find the visual feature of lip shapes Also research did by Ouloul [15] proposed a new algorithm that combines the RGB image with Depth map which is less sensible to illumination changes. Sulong [16] proposed a method to overcome the low image contrast, combining twoblock feature; mean of gradient magnitude and coherence, where the fingerprint image is segmented into background, foreground or noisy regions, has been done. Except for the noisy regions in the foreground, there are still such noises existed in the background whose coherences are low, and are mistakenly assigned as foreground.

In the developing countries such in Indonesia, availability of panoramic device is not evenly. Most of the equipment is only available in major hospitals in the provincial capital, while for the other cities are not always available. If there is, usually panoramic devices with low resolution. In this paper, we proposed to improve image quality produced by low-resolution panoramic device by implementing point processing method. It is a simple method and suitable for implementing in developing countries such in Indonesia. In 
our previous research [17]-[22] we focused our work on the implementation of digital image processing techniques in the development of technologies supporting health services in rural areas, such as early detection of cataracts as well as optimization of the low-resolution ultrasonography, therefore in this research we still keep emphasize on the optimization of health services in developing countries. In this case, we aim to improve the quality of low-resolution panoramic image so that people in developing countries can still be able to take advantage of the panoramic radiology diagnosis optimally.

\section{RESEARCH METHOD}

Digital image processing techniques is an image processing to produce images in accordance with our wishes or quality to be better. Image processing operations can be divided can be divided into operations that generate an output based on the value of a single pixel, the operation that produces output based on a collection of pixels adjacent to each other and the operation that produces output based on all the pixels in the image. Point processing method is part of an operation that generates output based on the value of a single pixel that covers thresholding, contrast stretching and gray level reduction [23].

\subsection{Pixel Operation}

In the image processing, there is a term operations pixels or sometimes called operations pixel-topixel. Operation of pixels is the image processing operation that maps each pixel relationship that relies on pixel itself. If $\mathrm{f}(\mathrm{y}, \mathrm{x})$ expressed the value of a pixel in the image of $\mathrm{f}$ and $\mathrm{g}(\mathrm{y}, \mathrm{x})$ expressed pixel processing results from $\mathrm{f}(\mathrm{y}, \mathrm{x})$, the relationship [20] can be expressed by Equation 1.

$$
g(y, x)=T(f(y, x))
$$

In this case, $\mathrm{T}$ stating the function or operation kind imposed on pixel $\mathrm{f}(\mathrm{y}, \mathrm{x})$. Operating model is what will be discussed in this paper.

\subsection{Brightness Increasing}

The basic operation is often performed on the image is the increase in brightness. This operation is necessary in order to make the image becomes brighter. Mathematically, the increased brightness is done by adding a constant to the value of the entire pixel [24]. For example, $f(y, x)$ expressed the pixel values in the gray scale image at coordinates $(\mathrm{y}, \mathrm{x})$. Thus, a new image becomes as expressed by Equation 2 .

$$
8(y, x)=f(y, x)+\beta
$$

A new image has increased the brightness value of all pixels on the image of the original large $\beta \mathrm{f}(\mathrm{y}, \mathrm{x})$. If the $\beta$ form of negative numbers, the brightness will decrease or become darker.

\subsection{Contrast Stretching}

The contrast in an image stating the distribution of light and dark shades of color. A gray-scale image is said to have a low contrast when the distribution of color tend to narrow the range of gray levels. Conversely, if the image has a high contrast range of gray levels distributed over wide. Contrast can be measured based on the difference between the value of the highest intensity and the lowest intensity values that make up the pixels in the image [24]. In order the distribution of pixel intensity changes necessary to stretch the contrast. This is done by using the formula as expressed by Equation 3 .

$$
g(y, x)=\alpha f(y, x)
$$

Based on the above formula, the contrast will increase if $\alpha>1$ and the contrast will suffer if $\alpha<1$.

\subsection{Brightness and Contrast Combination}

Operation for increasing brightness and contrast stretching can be performed simultaneously in order to improve image quality [24]. In general, a combination of the two operations can be expressed by Equation 4.

$$
g(y, x)=\alpha f(y, x)+\beta
$$


However, if we intend to make arrangements so that the gray level in the image of $f$ in the range of $\mathrm{f} 1$ and $\mathrm{f} 2$ be the image of $\mathrm{g}$ with cedar between $\mathrm{g} 1$ and $\mathrm{g} 2$, the required formula is expressed by Equation 5 .

$$
g(x, 2)-s_{1}+\left(\frac{g_{2}-g_{1}}{f_{2}-f_{1}}\right)\left[f\left\{y_{1}, x\right)-f_{2}\right]
$$

\subsection{Histogram Equalization}

Histogram equalization) provide a sophisticated method for modifying the dynamic range and contrast of an image by altering that image such that its intensity histogram has a desired shape. Unlike contrast stretching, histogram modeling operators may employ non-linear and non-monotonic transfer functions to map between pixel intensity values in the input and output images. Histogram equalization employs a monotonic, non-linear mapping which re-assigns the intensity values of pixels in the input image such that the output image contains a uniform distribution of intensities [25].

\section{RESULTS AND ANALYSIS}

The data used in this research are panoramic images taken from Dental Hospital Jenderal Soedirman University, Purwokerto. Examples of input image is shown in Figure 2.
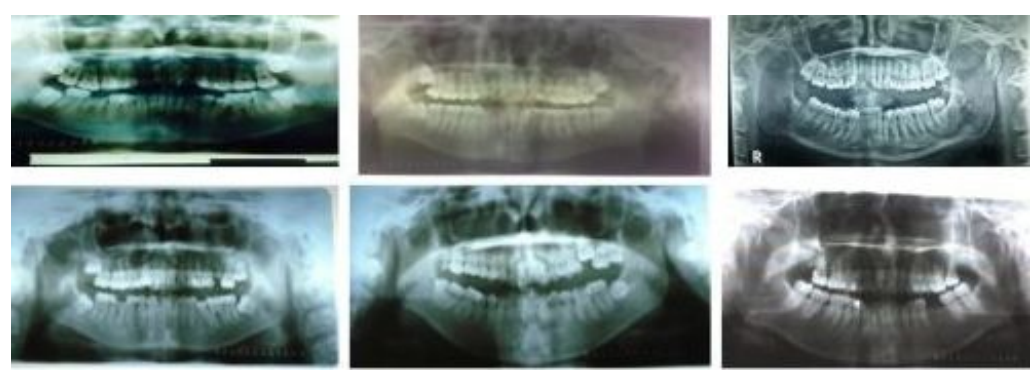

Figure 2. Examples of input image (source, Widodo [2])

In the process of improving image quality by using contrast stretching method, starting by reading input image as shown in Figure 2. The next step is to change the image size to reduce the computational load. Then in order to do contrast stretching process, the first color image transformed into a grayscale image. After getting a grayscale image, the next is to analyze the histogram of each input image as a based to calculate the value of R1, S1, R2 and S2. In our previous research [2], we did this analysis and we classified our images data into three classifications, there are tend to left, tend to right and tend to left and right After knowing the tendencies of each histogram, it can be done further analysis is to determine the value of the gray-R1, S1, R2, S2, A1, A2 and A3 as discussed in our previous results [2] and detailed results are described in Figure 3.

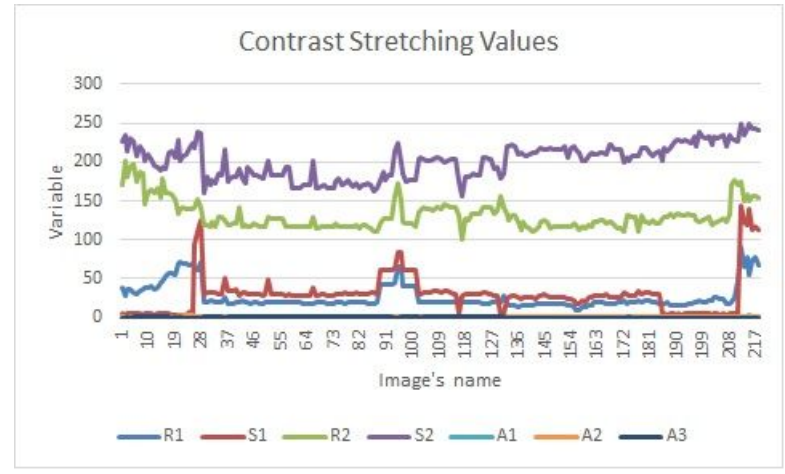

Figure 3. Calculation result of contrast stretching values 
According to the data obtained it appears there are some differences when testing input on any type of histogram as follows (1) For the category of bright histogram, have a spread of gray that dominates the right side, the input to $\mathrm{S} 1$ is smaller than $\mathrm{R} 1$, is caused by input $\mathrm{S} 1$ smaller can encourage changes in location of pixels new that leads to the left, so that the type of ideal histogram can be achieved. (2) For the category of dark histogram, have a spread of gray that dominates the left side, this was due to the input S1 larger so as to encourage a change of pixels new leads in the right direction so that the type of ideal histogram can be achieved by distribution of its gray values evenly from left to right. (3) For the category of evenly dark and bright histogram, have a gray spread fairly evenly shaped valley peak from left to right. Referring to the viewpoint of the input almost the same type of dark histogram. One of the things that distinguishes that in determining the estimated input gray value, which seek pixel intensity histogram at the center so that it can be seen clearly balance the spread of gray of the image histogram. After entering a value of gray in each image which totaled 218 panoramic image, the next step is doing for the main processes contrast stretching. For comparative analysis, we compared the results of grayscale histogram and contrast stretching histogram as described in Table 1.

Table 1. Example of Comparison Result between Grayscale Histogram and Contrast Stretching Histogram

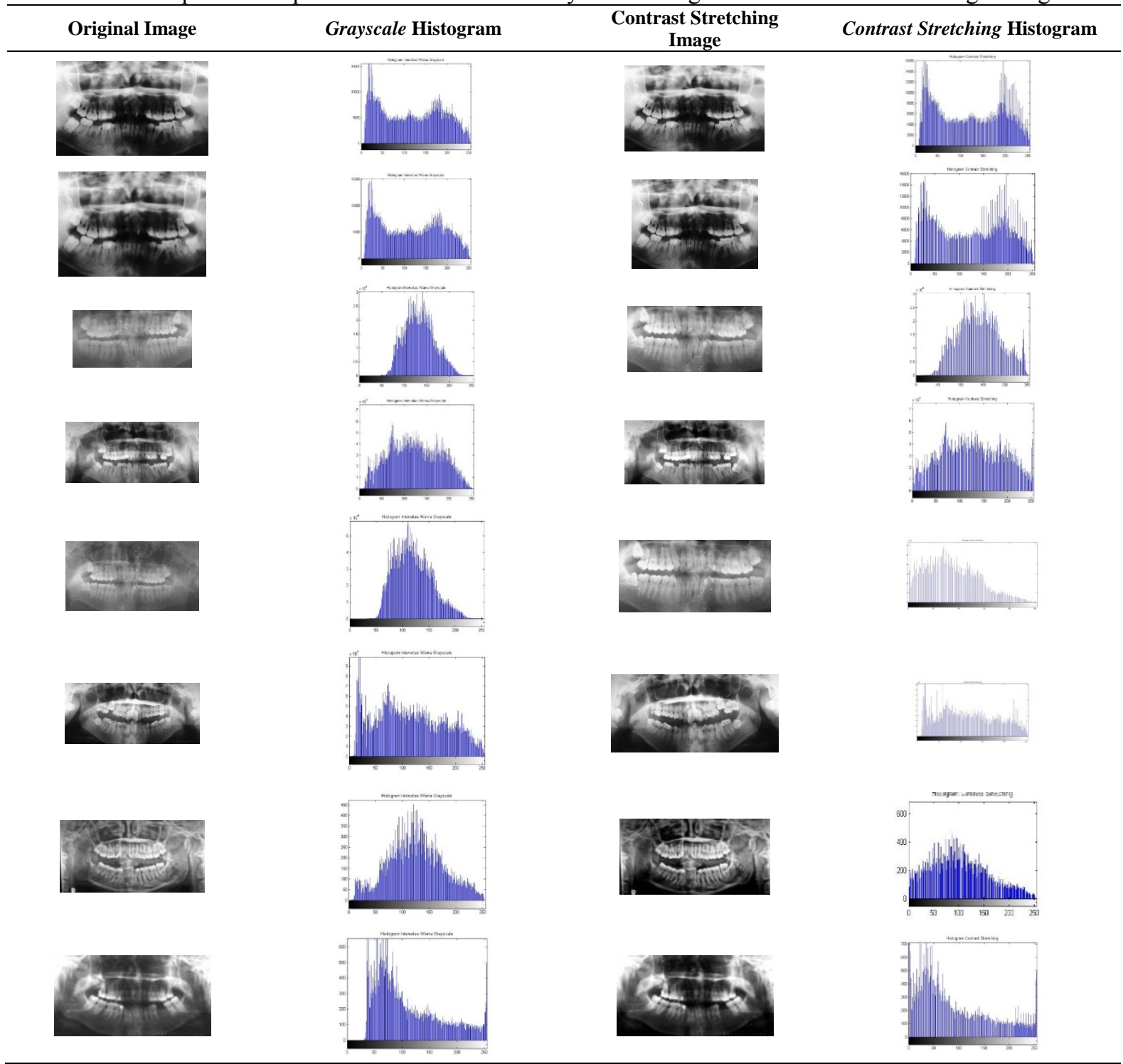

Contrast stretching method is a technique to get a new image with better contrast than the contrast of the original image. The idea of the contrast stretching process is to improve the dynamic field of gray level in the image to be processed. We conclude, contrast stretching method is a method of image enhancement by 
increasing the contrast of the image through several conditions. The condition is when the process of manipulating the pixels do not use the same operation on all histograms, but using some of the conditions, so that the current image settings can be more efficient because of some parts of the image there needs to be slightly enhanced brightness and there also needs to be much improved brightness.

In order to know characteristic of contrast stretching histogram, we took two samples of random images. Characteristics obtained by investigating differences location of the pixels in the grayscale histogram and contrast stretching histogram on each image that is presented in Figure 4.

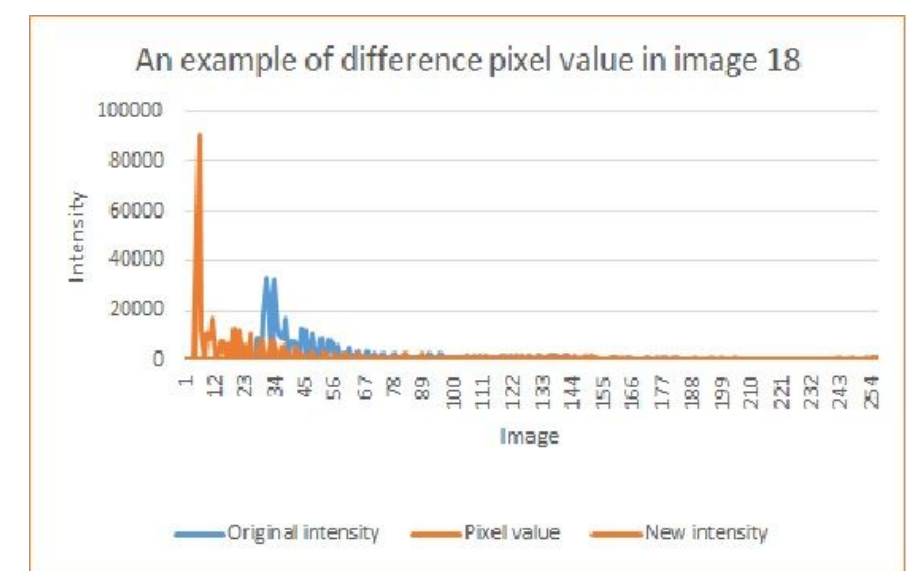

Figure 4. An example of difference pixel value in an image (Image 18, R1 = 33, S1 = 5, R2 = 198, S2=226)

According to Figure 4 using contrast stretching method there are three areas stretching namely A1 (first area), A2 (second area) and A3 (third area). The area is an area stretching scaling which is based on the basic equation contrast stretching. In mathematics, the area shows the value of a straight line gradient. If the gradient of a straight line generating value $<1$ then the new pixel area will be narrowed area of pixels, and the merger intensity values of the original pixels. Instead gradient a straight line $>1$ there will be a widening or stretching the pixel area. Improving image quality using Contrast Stretching method does not change the value of the intensity of an image.

Based on the formulation of the problem that we made previously about optimization of the grayscale image causing some limitations in the research problem. It showed in the research is still focused on a narrow scope and there are still many shortcomings. Especially on the grayscale values R1, S1, R2 and $\mathrm{S} 2$ which has no reference values. In other words, there are no parameters or reference to whether the value of an estimated input by the user generating the panoramic image output more clearly or not. Based on these problems, we are testing the hypothesis in the form of 'second opinion' to the dental hospital Purwokerto by attaching the data of the original image and the new image of the results of our research. In practice, the radiological merely compare and choose the one image of the original image with a new image. Therefore, it is expected through hypothesis testing in the form of 'second opinion' that can justify the results of our testing. Hypothesis testing based on Sugiyono [26] is comprised of two types: (1) The null hypothesis (H0) is the hypothesis that a lack of correlation between the independent variable (X) and the dependent variable (Y). (2) The working hypothesis (H1) is the hypothesis that the relationship between the independent variable $(\mathrm{X})$ and the dependent variable (Y) being investigated. The calculation results H1, will be used as the basis of research data search. Based on the hypothesis test reports, it turns out H1 (working hypothesis) "acceptable" i.e. "There is a significant effect of the contrast stretching methods on the quality of the panoramic image". It has been proven by the fact from the number of new images that have been more than the original image that are: (i) the original image of a selected number of 35 pieces. (ii) The new image of a selected number of 183 pieces. It means is $83.9 \%$ successful image. While $16.1 \%$ declared unfit image according to the radiology. It is caused by some following reasons: (1) there is a linguistic information from several panoramic images are reduced. Whereas in the medical world, the information is really important, as a reference to diagnose the patient's condition. (2) Visually, the boundary area of an area of several panoramic images are less clear and unequivocal. This is because the contrast is too high thus affecting boundary or affirmation of an area. 


\section{CONCLUSION}

Based on the deployment of pixels, histogram panoramic image can be grouped into three groups; (i) for the type of bright histogram, the spread of gray values dominated in the right side. Therefore the value of $\mathrm{S} 1$ is smaller than R1. (ii) For the type of dark histogram, the spread of gray values dominated in the left side. Therefore the value of S1 is greater than R1. (iii) For the type of evenly dark and bright histogram, the spread of gray values evenly from left to right, where the input value is almost equal to that dark histogram S1 greater than R1. However, the range between R1 to S1 is greater than the dark histogram. R2 value must be greater than S2 because a reference point on the left, therefore the stretch should lead to right, it applies both to dark, bright or evenly dark and bright. After improving image quality, about 8 samples representing 218 images, indi-cating a significant improvement towards a better image quality. It is based on a better visual and new image histogram has been evenly spread. If an area or a gradient of a straight line generating value smaller than 1 then the new pixel area will be narrowed and the merger intensity values of the original pixels. Conversely, if an area or gradient a straight line greater than 1 there will be a widening or stretching the pixel area. Improving image quality using Contrast Stretching method does not change intensity values of an image. According to the second opinion from Dental Hospital Jenderal Soedirman University, performance of our method is $83.9 \%$. While about $16.1 \%$ failed due to linguistic information and visual boundary area.

\section{ACKNOWLEDGEMENTS}

We would like to thanks very much to Dental Hospital Jenderal Soedirman University Puwokerto for panoramic image data. This research is supported by Dana BLU Jenderal Soedirman University through Hibah Unggulan Unsoed.

\section{REFERENCES}

[1] D. J. Iannucci and R. M. L. J. Howerton, "Dental Radiography," 4th ed. United States, Elsevier, 2012.

[2] H. B. Widodo, et al., "Calculating Contrast Stretching Variables in Order to Improve Dental Radiology Image Quality," in International Conference on Engineering and Technology for Sustainable Development (ICET4SD), 2015.

[3] B. Molander, et al, "Image Quality in Panoramic Radiography," Dentomaxillofac Radiol., vol/issue: 24(1), pp. 1722, 1995

[4] V. Rushton, et al., "Screening panoramic radiology of adults in general dental practice: Radiological findings," Br. Dent. J., vol/issue: 190(9), pp. 495-501, 1995.

[5] J. G. Chaffin, et al., "Validity of using a panoramic radiograph for initial dental classification of army recruits," Mil. Med., vol/issue: 169(5), pp. 368-372, 2004.

[6] A. Takahashi, et al., "Localizing the mandibular canal on dental CT reformatted images: Usefulness of panoramic views," Surg. Radiol. Anat., vol/issue: 35(9), pp. 803-809, 2013.

[7] A. S. Suomalainen, et al., "Dentomaxillofacial imaging with panoramic views and cone beam CT.," Insights Imaging, vol/issue: 6(1), pp. 1-16, 2015.

[8] Ö. S. Sezgin, et al., "Comparative dosimetry of dental cone beam computed tomography, panoramic radiography, and multislice computed tomography," Oral Radiol., vol/issue: 28(1), pp. 32-37, 2012.

[9] E. T. Ertas, et al., "Incidental findings of carotid artery stenosis detected by calcifications on panoramic radiographs: Report of three cases.," Oral Radiol., vol/issue: 26(2), pp. 116-121, 2010.

[10] M. A. Rotondi and A. Donner, "A confidence interval approach to sample size estimation for interobserver agreement studies with multiple raters and outcomes," J. Clin. Epidemiol., vol/issue: 65(7), pp. 778-784, 2012.

[11] K. Pan-Fu, et al., "Fully Automatic Abdominal Fat Segmentation System from a Low Resolution CT Image," J. Comput., vol/issue: 26(2), pp. 64-77, 2015.

[12] H. H. Chang, et al., "A High Payload Steganography Scheme for Color Images Based on BTC and Hybrid Strategy," J. Comput., vol/issue: 26(2), pp. 46-55, 2015.

[13] S. Gopinathan, et al., "Wavelet and FFT Based Image Denoising Using Non-Linear Filters," Int. J. Elec, vol/issue: 5(5), 2015.

[14] B. A. Faridah and B. Listyana S., "Lip image feature extraction utilizing snake's control points for lip reading applications," Int. J. Electr. Comput. Eng., vol/issue: 5(4), pp. 720-728, 2015.

[15] M. I. Ouloul, et al., "An Efficient Face Recognition Using SIFT Descriptor in RGB-D Images," Int. J. Electr. Comput. Eng., vol/issue: 5(6), pp. 1227-1233, 2015.

[16] G. Sulong, "Segmentation of Fingerprint Image Based on Gradient Magnitude and Coherence," Int. J. Electr. Comput. Eng., vol/issue: 5(5), pp. 1202-1215, 2015.

[17] R. Supriyanti, et al., "A Simple Screening for High-Risk Pregnancies in Rural Areas Based Expert System," Telkomnika, vol/issue: 13(2), pp. 661-669, 2015.

[18] R. Supriyanti, et al., "Separability filter for localizing abnormal pupil: Identification of input image," Telkomnika, vol/issue: 11(4), pp. 783-790, 2013.

[19] R. Supriyanti, et al., "Comparing edge detection methods to localize uterus area on ultrasound image," in Proc. of 2013 3rd Int. Conf. on Instrumentation, Communications, Information Technol., and Biomedical Engineering: 
Science and Technol. for Improvement of Health, Safety, and Environ., ICICI-BME 2013, pp. 152-155, 2013.

[20] R. Supriyanti, et al., "Extracting appearance information inside the pupil for cataract screening," in Proceedings of the 11th IAPR Conference on Machine Vision Applications, pp. 342-345, 2009.

[21] R. Supriyanti, et al., "A simple and robust method to screen cataracts using specular reflection appearance," in Proc. SPIE 6915 Medical Imaging, 2008.

[22] R. Supriyanti, et al., "Compact Cataract Screening System: Design and Practical data Acquisition," in International Conference on Instrumentation, Communication, Information Technology and Biomedical Engineering (ICICI-BME), 2009.

[23] B. Willkinson and M. Allen, "Parallel Programming: Techniques and Application Using Networked Workstations and Parallel Computers," Second Edi. New Jersey: Pearson Prentice Hall, 2005.

[24] A. Kadir and A. Soesanto, "Teori dan Aplikasi Pengolahan Citra," Yogyakarta Indonesia: Penerbit Andi, 2013.

[25] R. Fisher, et al., "Histogram Equalization," 2003. [Online]. Available: http://homepages.inf.ed.ac.uk/rbf/HIPR2/histeq.htm.

\section{BIOGRAPHIES OF AUTHORS}

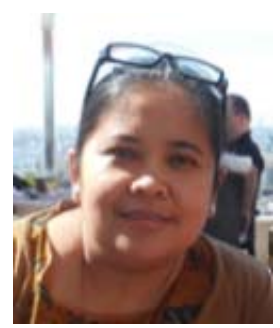

Retno Supriyanti is an academic staff at Electrical Engineering Department,Jenderal Soedirman University, Indonesia. She received her PhD in March 2010 from Nara Institute of Science and Technology Japan. Also, she received her M.S degree and Bachelor degree in 2001 and 1998, respectively, from Electrical Engineering Department, Gadjah Mada University Indonesia. Her research interests include image processing, computer vision, pattern recognition, biomedical application, e-health, tele-health and telemedicine.

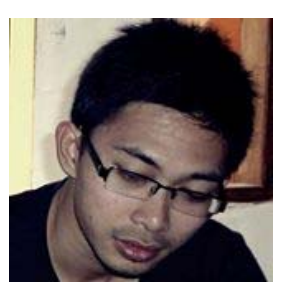

Ariep Soelaiman Setiadi received his Bachelor degree from Electrical Engineering Depratment, Jenderal Soedirman University Indonesia. His research interest is Decision Support System field.

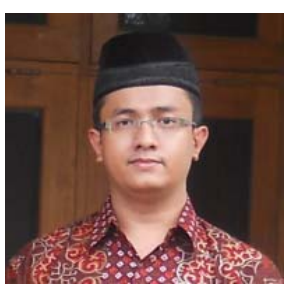

Yogi Ramadhani is an academic staff at Electrical Engineering Department, Jenderal Soedirman University, Indonesia. He received his MS Gadjah Mada Universirt Indonesia, and his Bachelor degree from Jenderal Soedirman University Indonesia. His research interest including Computer Network, Decision Support Syetem, Telemedicine and Medical imaging

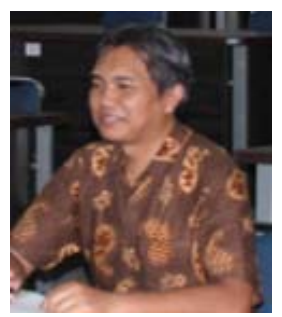

Haris Budi Widodo is an academic staff at Public Health Department, Jenderal Soedirman University, Indonesia. He received his Ph.D from Airlangga University Indonesia. Also He received his M.S degree and bachelor degree from Gadjah Mada University Indonesia. His research interest including public health, e-health and telemedicine 